\title{
Communications
}

\section{Dialog Ethik}

\section{Kindeswohl im Spital - was hospitalisier- ten Kindern und Jugendlichen gut tut}

Die Studie «Kindeswohl im Spital - eine qualitative Studie zum Wohlbefinden hospitalisierter Kinder und Jugendlicher» der Stiftung Dialog Ethik und des Kinderspitals Zürich präsentiert einen Kriterienkatalog für die Behandlung und Betreuung von Kindern und Jugendlichen im Spital.

In den letzten drei Jahrzehnten hat sich die Situation für Kinder und Jugendliche in Schweizer Kinderspitälern stark verändert. Während Kinder früher beispielsweise die meiste Zeit im Bett verbringen mussten und ihre Eltern nur wenige Stunden pro Tag sehen durften, hat sich auf der Grundlage der modernen Kinderrechte der Alltag im Kinderspital verbessert. Auch vor dem Hintergrund der hochspezialisierten Medizin hat sich das Kindeswohl zu einem zentralen Konzept entwickelt. Was aber ist mit «Kindeswohl» gemeint?

Diese Frage stellten wir im Rahmen der Studie Patienten, Eltern und zahlreichen Mitarbeitenden des Kinderspitals Zürich und des Rehabilitationszentrums Affoltern (Ärzte, Pflegende, administrative Mitarbeitende,
Clowns, Freiwillige, Lehrpersonen, Physiotherapeuten, Psychologen, Sozialberater etc.). Auf der Grundlage der insgesamt 68 Interviews wurden zu den folgenden Kategorien 40 Kriterien für das Kindeswohl im Spital erarbeitet:

- Krankheits- und therapiebezogene Bedürfnisse des Kindes

- emotionales Wohlbefinden des Kindes

- Ermutigung und Befähigung des Kindes

- Normalität und gewohntes Leben für das Kind

- Familienorientierung

- professionelle Betreuung

Dieser Kriterienkatalog dient als Orientierung in der Praxis und kann in Entscheidungen im Einzelfall einfliessen oder bei der Reflexion über die Rahmenbedingungen für die Realisierung des Kindeswohls im Spital einbezogen werden.

Der Studienbericht kann auf der Website von Dialog Ethik heruntergeladen werden: www.dialog-ethik.ch

Ansprechpartner:

Dr. phil. Andrea Abraham, Leitung Forschung Institut Dialog Ethik Tel.: 0442524201

E-Mail: aabraham[at]dialog-ethik.ch
Caisse de secours des médecins suisses

\section{Contributions reçues}

Du $1^{\text {er }}$ avril au 30 juin 2016, nous avons reçu 22 dons d'une valeur totale de 3855.17 francs.

Le Conseil de fondation de la Caisse de secours des médecins suisses est très content de pouvoir publier ces chiffres et tient à remercier vivement tous les donateurs. Afin que les contributions puissent être versées entièrement aux destinataires, nous avons pris la décision de renoncer à l'envoi de lettres de remerciement pour les dons inférieurs à 500 francs. Nous espérons que les donateurs feront preuve de compréhension et nous leur adressons encore une fois nos remerciements.

Le trésorier du Conseil de fondation 